\title{
Prevalência de helmintos intestinais em três mesorregiões do Estado de Minas Gerais
}

\author{
Prevalence of intestinal helminths in three \\ regions of Minas Gerais State
}

\begin{abstract}
Omar dos Santos Carvalho', Henrique Leonardo Guerra², Yoná Rose Campos', Roberta Lima Caldeira ${ }^{1}$ e Cristiano Lara Massara ${ }^{1}$
\end{abstract}

\begin{abstract}
Resumo Foi realizado um levantamento das helmintoses intestinais em 18.973 escolares do primeiro grau (7 a 14 anos), da rede pública do Estado de Minas Gerais, utilizando o método de Kato-Katz de exame de fezes (duas lâminas por amostra). Foram estudadas 3 mesorregiões: Triângulo Mineiro/Alto Paranaíba (60 municípios), Noroeste de Minas (13) e Sul/Sudoeste (144). Entre os escolares examinados, 15.545 (82\%) estavam negativos, $2.863(15 \%)$ monoparasitados e $565(3 \%)$ poliparasitados. A prevalência de A. lumbricoides foi de $10,3 \%$, de T. trichiura 4,7\%, de ancilostomídeos $2,9 \%$, de $\mathrm{E}$. vermicularis $1,2 \%$, de $\mathrm{H}$. nana $0,4 \%$ e de Taenia sp 0,2\%. As maiores prevalências de helmintos/mesorregião foram de 24,2\% para T. trichiura e 18,7\% para A. lumbricoides (Sul/Sudoeste) e 12,1\% para ancilostomídeos e 0,7\% para Taenia sp (Noroeste de Minas). As helmintoses intestinais continuam sendo um grave problema de saúde pública, inclusive em áreas onde as condições socioeconômicas são mais favoráveis.
\end{abstract}

Palavras-chaves: Helmintos intestinais. Prevalência. Estado de Minas Gerais.

Abstract A parasitological assay of feces, through the Kato-Katz method, was undertaken in 18,973 schoolchildren (7 to 14 years old), at primary school level, from a public school of the State of Minas Gerais. Three mesoregions were studied: Triângulo Mineiro/ Alto Paraíba (60 municipalities); northwest Minas Gerais (13) and south/south-west regions of Minas Gerais (144). Among the examined children, 15,545 (82\%) were negative; 2,863 (15\%) were infected with a single species of helminthes and $565(3 \%)$ were infected by more than one species. The prevalence rates were: A. lumbricoides 10.3\%; T. trichiura 4.7\%; hookworm 2.9\%; E. vermicularis $1.2 \% ; \mathrm{H}$. nana $0.4 \%$ and Taenia sp $0.2 \%$. The largest helminthes prevalence/mesoregion were for T. trichiura (24.2\%) and A. lumbricoides (18.7\%) in the south/south-west region; hookworm (12.1\%) and Taenia $\mathrm{sp}(0.7 \%)$ in the northwest region of Minas Gerais. Intestinal helminthiasis is still regarded as a serious public health problem, including regions where the socio-economic conditions are more favorable.

Key-words: Intestinal helminthes. Prevalence. State of Minas Gerais.

De um modo geral, as informações sobre a prevalência de helmintos intestinais no Brasil são escassas ou mesmo nulas para determinadas regiões. Quando existe, esta informação é fragmentada, desatualizada e as técnicas parasitológicas utilizadas não são coincidentes, impedindo a comparação de dados. Em 1950, Pellon \& Teixeira ${ }^{9}$ realizaram o Inquérito Nacional da Esquistossomose em 11 estados brasileiros, utilizando o método de sedimentação das fezes. Foram examinadas 440.784 amostras de fezes de escolares de 7 a 14 anos em todo o Brasil e $162.176 \mathrm{em}$ Minas Gerais. Em $1953^{10}$, os mesmos autores realizaram, também pelo método de sedimentação, o Inquérito
Helmintológico Escolar em 313 localidades de cinco estados brasileiros - Rio de Janeiro, Paraná, Santa Catarina, Goiás e Mato Grosso, examinando 174.192 escolares. Posteriormente, Campos \& Briques ${ }^{1}$ realizaram um Levantamento Multicêntrico de Parasitoses Intestinais, em 10 estados, onde foram examinadas 18.151 amostras de fezes de escolares de 7 a 14 anos, pelo método de KatoKatz, (uma lâmina por amostra) abrangendo 16 centros universitários. No presente trabalho foi realizado um levantamento da prevalência das helmintoses intestinais em 3 mesorregiões do Estado de Minas Gerais, consideradas indenes para esquistossomose.

\footnotetext{
1. Laboratório de Helmintoses Intestinais do Centro de Pesquisas René Rachou da Fundação Oswaldo Cruz, Belo Horizonte, MG. 2. Laboratório de Epidemiologia e Antropologia Médica do Centro de Pesquisas René Rachou da Fundação Oswaldo Cruz, Belo Horizonte, MG.

Endereço para correspondência: Dr. Omar dos Santos Carvalho. Centro de Pesquisas René Rachou/FIOCRUZ. Av. Augusto de Lima 1715, Barro Preto, 30190-002 Belo Horizonte, MG.

Tel 5531 3295-3566, Fax 3295- 3115.

e-mail:omar@cpqrr.fiocruz.br

Recebido para publicação em 5/11/2001.
} 


\section{MATERIAL E MÉTODOS}

Foi realizado um levantamento parasitológico de fezes pelo método de Kato-Katz ${ }^{5}$, examinando-se duas lâminas por amostra, em 18.973 escolares do primeiro grau (7 a 14 anos) da rede pública do Estado de Minas Gerais. Foram estudadas três mesorregiões: Triângulo Mineiro/Alto Paranaíba (60 municípios), Noroeste de Minas (13) e Sul/Sudoeste (144) (Figura 1). A prevalência foi calculada tendo como numerador, o número de estudantes com exame positivo para cada uma das parasitoses estudadas e como denominador, o número de estudantes examinados. O intervalo de confiança de $95 \%$ das estimativas de prevalência foi calculado segundo a fórmula $1,96\left(p(1-p) / n^{0,5}\right.$. O cálculo dos indivíduos monoparasitados, poliparasitados e as associações de infecção foram feitos utilizando o programa estatístico EPI-info.

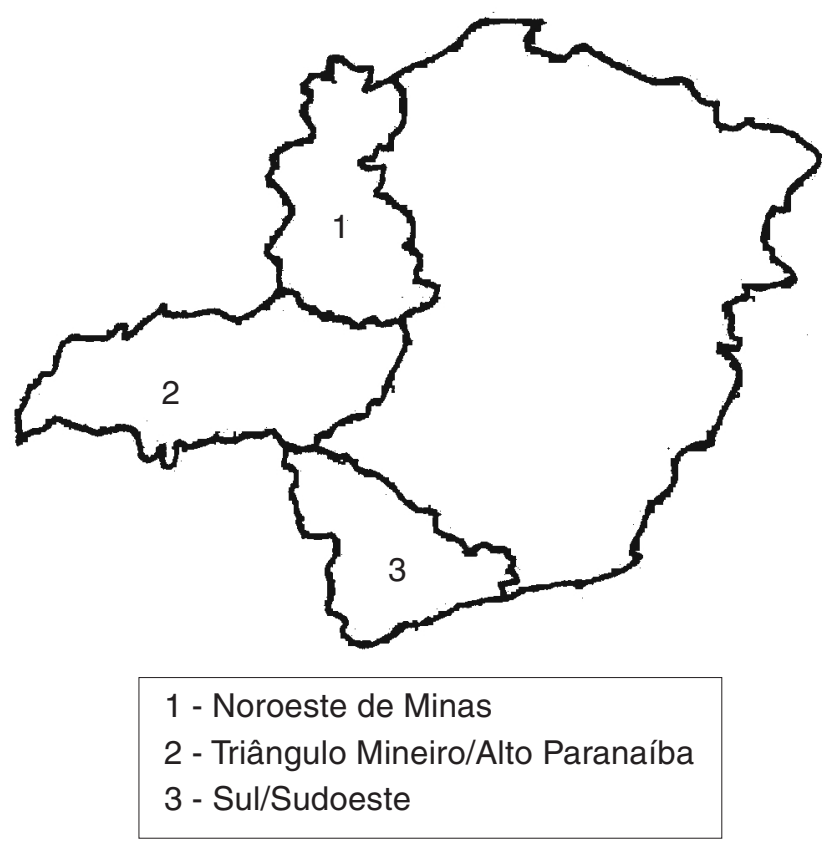

Figura 1 - Mapa de Estado de Minas Gerais mostrando em destaque as mesorregiões.

\section{RESULTADOS}

Entre os 18.973 escolares examinados, $3.428(18,1 \%)$ encontravam-se parasitados, sendo $1.804(52,6 \%)$ do sexo masculino e $1.624(47,4 \%)$ do sexo feminino (Tabela 1). Do total de escolares examinados, $1.963(10,3 \%)$ estavam positivos para Ascaris lumbricoides, $886(4,7 \%)$ para Trichuris trichiura, $561(2,9 \%)$ para ancilostomídeos, $230(1,2 \%)$ para Enterobius vermicularis, $73(0,4 \%)$ para Hymenolepis nana e $35(0,2 \%)$ para Taenia sp. Os resultados dos exames de fezes por meso e microrregião podem ser vistos na Tabela 2. As maiores prevalências, por microrregião foram de $24,2 \%$ para $T$. trichiura e $18,7 \%$ para A. lumbricoides (microrregião de São Lourenço - mesorregião Sul/Sudoeste), 12,1\% para ancilostomídeos (microrregião de Unaí - mesorregião Noroeste de Minas), 2,8\% para E. vermicularis (microrregião de Patos de Minas - mesorregião do Triângulo/Alto Paranaíba), 1,3\% para $H$. nana (microrregião de Paracatu e Araxá mesorregião Noroeste de Minas e Triângulo/Alto Paranaíba, respectivamente) e 0,7\% para Taenia sp (microrregião de Unai - mesorregião Noroeste de Minas). Do total de escolares infectados, 2.863 (83,5\%) apresentavam-se com um parasita, $537(15,6 \%)$ com dois parasitas e $28(0,9 \%)$ com três parasitas (Tabela 3$)$.

\section{DISCUSSÃO}

Os helmintos intestinais constituem um grave problema de saúde pública em diversas regiões do mundo. Sua presença está associada, quase sempre, ao baixo desenvolvimento econômico, carência de saneamento básico e falta de higiene. As infecções helmínticas são consideradas uma das principais 
Tabela 1 - Distribuição da infecção por helmintos intestinais, por sexo, em três mesorregiões do Estado de Minas Gerais

\begin{tabular}{|c|c|c|c|c|c|c|}
\hline \multirow[b]{2}{*}{ Sexo } & \multicolumn{2}{|c|}{ Total } & \multicolumn{2}{|c|}{ Negativos } & \multicolumn{2}{|c|}{ Positivos } \\
\hline & $n^{\circ}$ & $\%$ & $n^{\circ}$ & $\%$ & $n^{\circ}$ & $\%$ \\
\hline Masculino & 9.462 & 49,9 & 7.658 & 49,3 & 1.804 & 52,6 \\
\hline Feminino & 9.511 & 50,1 & 7.887 & 50,7 & 1.624 & 47,4 \\
\hline Total & 18.973 & 100,0 & 15.545 & 100,0 & 3.428 & 100,0 \\
\hline
\end{tabular}

Tabela 2 - Prevalência de helmintoses intestinais em escolares de $1^{\circ}$ grau em três mesorregiões do Estado de Minas Gerais.

\begin{tabular}{|c|c|c|c|c|c|c|c|}
\hline \multicolumn{8}{|l|}{ Mesorregiões/ } \\
\hline Microrregiões & Exames realizados & A. lumbricoides & T. trichiura & Ancilostomídeos & Taenia sp & H. nana & E. vermicularis \\
\hline Noroeste de Minas & 3.430 & 8,1 & 0,9 & 8,6 & 0,4 & 1,0 & 1,4 \\
\hline Paracatu & 2.200 & 9,6 & 1,0 & 6,6 & 0,2 & 1,3 & 1,5 \\
\hline Unai & 1.230 & 5,5 & 0,7 & 12,2 & 0,7 & 0,4 & 1,3 \\
\hline Triângulo/Alto Paranaíba & 11.325 & 13,3 & 3,5 & 2,0 & 0,1 & 0,3 & 1,2 \\
\hline Araxá & 1.226 & 18,1 & 5,2 & 1,2 & 0,1 & 1,3 & 1,9 \\
\hline Frutal & 503 & 2,8 & 0,6 & 2,6 & 0,2 & 0,0 & 0,6 \\
\hline Ituiutaba & 510 & 3,7 & 0,4 & 4,1 & 0,0 & 0,4 & 1,2 \\
\hline Patos de Minas & 1.824 & 15,1 & 2,3 & 3,0 & 0,2 & 0,5 & 2,8 \\
\hline Patrocínio & 1.156 & 11,5 & 4,1 & 2,9 & 0,1 & 0,1 & 1,5 \\
\hline Uberaba & 3.174 & 16,3 & 5,5 & 0,7 & 0,2 & 0,2 & 0,7 \\
\hline Uberlândia & 2.932 & 10,8 & 2,4 & 2,5 & 0,1 & 0,2 & 0,6 \\
\hline Sul/Sudoeste & 4.218 & 10,5 & 10,8 & 0,8 & 0,2 & 0,0 & 0,1 \\
\hline Alfenas & 393 & 8,7 & 7,9 & 0,0 & 0,3 & 0,0 & 2,0 \\
\hline Andrelândia & 284 & 15,1 & 15,1 & 1,4 & 0,0 & 0,0 & 1,8 \\
\hline Itajubá & 379 & 17,4 & 15,0 & 0,8 & 0,3 & 0,0 & 1,3 \\
\hline Passos & 432 & 3,7 & 3,2 & 1,2 & 0,2 & 0,0 & 0,9 \\
\hline Poços de Caldas & 414 & 6,8 & 7,2 & 0,5 & 0,0 & 0,0 & 0,2 \\
\hline Pouso Alegre & 533 & 12,8 & 11,7 & 1,0 & 0,4 & 0,0 & 0,8 \\
\hline São Sebastião Paraíso & 507 & 6,1 & 7,5 & 1,6 & 0,2 & 0,0 & 0,8 \\
\hline São Lourenço & 327 & 18,7 & 24,2 & 0,3 & 0,0 & 0,0 & 1,8 \\
\hline Sta Rita do Sapucaí & 372 & 9,9 & 12,6 & 0,8 & 0,0 & 0,0 & 0,5 \\
\hline Varginha & 577 & 10,4 & 9,2 & 1,0 & 0,3 & 0,0 & 0,3 \\
\hline Total & 18.973 & 10,3 & 4,7 & 2,9 & 0,2 & 0,4 & 1,2 \\
\hline
\end{tabular}

Tabela 3 - Distribuição do número de helmintos intestinais em escolares de três mesorregiões do Estado de Minas Gerais.

\begin{tabular}{|c|c|c|c|c|c|c|c|c|}
\hline \multirow[b]{3}{*}{ Mesorregiões } & & & \multicolumn{6}{|c|}{ Parasitas } \\
\hline & \multicolumn{2}{|c|}{ Total de escolares parasitados } & \multicolumn{2}{|c|}{ mesor 1} & \multicolumn{2}{|c|}{ mesor 2} & \multicolumn{2}{|c|}{ mesor 3} \\
\hline & $\mathrm{n}^{\circ}$ & $\%$ & $\mathrm{n}^{\circ}$ & $\%$ & $\mathrm{n}^{\circ}$ & $\%$ & $\mathrm{n}^{\circ}$ & $\%$ \\
\hline Noroeste de Minas & 644 & 18,8 & 590 & 17,2 & 51 & 1,5 & 3 & 0,1 \\
\hline Triângulo/Alto Paranaíba & 2.045 & 59,7 & 1.763 & 15,6 & 272 & 2,4 & 10 & 0,5 \\
\hline Sul/Sudoeste & 739 & 21,5 & 510 & 12,1 & 214 & 5,1 & 15 & 0,4 \\
\hline Total & 3.428 & & 2.863 & & 537 & & 28 & \\
\hline
\end{tabular}

causas de morbidade nos escolares dos países em desenvolvimento, atingindo índices de até $90 \%{ }^{23}$. Apesar destas constatações os dados mais abrangentes de que dispomos até hoje, para o Brasil, são os de Pellon \& Teixeira ${ }^{910}$, onde foram realizados exames de fezes em 614.976 escolares de 16 estados, compreendendo as regiões Nordeste, Sudeste, Sul, e Centro Oeste. Campos \& Briques ${ }^{1}$ examinaram 5.360 escolares (7 a 14 anos) no Estado de Minas Gerais. A maior parte dos examinados encontrava-se positiva para
A. Iumbricoides $(59,5 \%)$, T. trichiura $(36,6 \%)$ e ancilostomídeos (2,6\%).

O espectro parasitário e a prevalência variam nas diferentes regiões, de acordo com as diferenças climáticas, sócio-econômicas, educacionais e condições sanitárias de cada área. Ludwig et $\mathrm{al}^{6}$ estabeleceram uma correlação entre as condições de saneamento básico e a freqüência de parasitoses, num período de 2 anos, no município de Assis (SP). Observaram também uma diminuição da prevalência das parasitoses 
intestinais com o aumento do número de ligações de água e esgoto, evidenciando uma relação inversa entre prevalência de exames positivos e população atendida por melhorias de saneamento básico. Mello et $\mathrm{al}^{7}$ realizaram um inquérito coproscópico, pelo método de sedimentação, em 86.157 amostras de fezes no Estado de Rondônia. Verificaram que $89,7 \%$ delas estavam positivas pelo menos para uma espécie de parasita e $42 \%$ poliparasitados. Observaram, ainda, que a faixa etária abaixo de 5 anos era a mais parasitada.

Nas três mesorregiões estudadas, a prevalência para os helmintos intestinais foi de $18 \%$. Pellon \& Teixeira, em $1950^{\circ}$, encontraram $89,4 \%$ de prevalência e Campos \& Briques, em 1988 1 , 44,2\%, ambas em Minas Gerais. Apesar de não serem passíveis de comparação, devido à utilização de diferentes técnicas coproscópicas e de cálculo de amostragem, podemos especular que esta diferença pode ser atribuída ao desenvolvimento das cidades estudadas que com o passar do tempo, proporcionaram melhores condições de vida para seus habitantes, além da disponibilidade de drogas de fácil administração.

Segundo Stephenson et al ${ }^{11}$ A. lumbricoides, T. trichiura e os ancilostomídeos (Necator americanus e Ancylostoma duodenale) são os helmintos mais freqüentemente encontrados em inquéritos helmintológicos.
Morales et $a^{8}$ examinaram pela técnica de Kato-Katz, 113.254 indivíduos em 100 municípios da Venezuela, observando uma prevalência de $32,6 \%$ para T. trichiura, $26,9 \%$ para $A$. lumbricoides e $5,6 \%$ para ancilostomídeos.

Podemos observar que nossos resultados estão de acordo com estes dados, pois dos helmintos estudados, as maiores prevalências foram constatadas para $A$. lumbricoides $(10,3 \%)$ e $T$. trichiura $(4,7 \%)$ em 19 microrregiões e ancilostomídeos (2,9\%) em 18. Além disso, quando comparamos as prevalências desses helmintos, observamos que $68,4 \%$ das microrregiões possuem as maiores prevalências para A. lumbricoides, $26 \%$ para $T$. trichiura e $5 \%$ para ancilostomídeos.

Concluímos pela necessidade de informações, em nível nacional, sobre as prevalências dos diversos helmintos, uma vez que as existentes são precárias e isoladas. Os programas de controle existentes em algumas regiões, como o tratamento com drogas e obras sanitárias trazem benefícios. Entretanto, apesar de imprescindíveis, esses esforços são, muitas vezes, ineficazes, pois constata-se a total falta de projetos educativos com a participação da comunidade. Portanto, deve-se considerar que o sucesso dos programas de controle depende da interação de melhoria das condições sócio-econômicas, infra-estrutura, educação em saúde e do engajamento comunitário.

\section{AGRADECIMENTO}

Agradecemos a José Geraldo Amorim da Silva pela leitura das lâminas.

\section{REFERÊNCIAS BIBLIOGRÁFICAS}

1. Campos R, Briques W. Levantamento multicêntrico de parasitoses intestinais no Brasil. Os resultados finais. Rhodia, São Paulo, 1988.

2. Cherter L, Cabeça M, Catapani WR Parasitoses Intestinais. Revista Brasileira e Medicina 51:126-132, 1995.

3. Cooper PJ, Guevara AE, Guderian RH. Intestinal helminthiasis in Ecuador: the relationship between prevalence, genetic, and socioeconomic factors. Revista da Sociedade Brasileira de Medicina Tropical 26:175-180, 1993.

4. Fleiss JL. Statistical Methods for rates and proportions. $2^{\text {nd }}$ Edition. John Wiley \& Sons, Inc, New York USA, 1981.

5. Katz N, Chaves A, Pellegrino J. A simple device for quantitative stool thick-smear technique in schistosomiasis mansoni. Revista do Instituto de Medicina Tropical 14:397-400, 1972.

6. Ludwig MK, Frei F, Alvares Filho A, Ribeiro-Paes JT. Correlação entre condições de saneamento básico e parasitoses intestinais na população de Assis, Estado de São Paulo. Revista da Sociedade Brasileira de Medicina Tropical 32:547-555, 1999.

7. Mello JR, Pinto RB, Salvajolli SR, Velasco MI. Distribuição geográfica das geohelmintoses em Rondônia In: Programas e
Resumos do XXXVII Congresso da Sociedade Brasileira de Medicina Tropical, Salvador, p 36, 2001.

8. Morales GR, Morales LP, Arteaga C, Martinella L, Rojas H. Prevalencias de las geohelmintiasis intestinales em 100 municípios de Venezuela (1989-1992). Revista da Sociedade Brasileira de Medicina Tropical 32:263-270, 1999.

9. Pellon AB, Teixeira I. Distribuição da esquistossomose mansônica no Brasil. Rio de Janeiro, Divisão da Organização Sanitária, 1950.

10. Pellon AB, Teixeira I. O inquérito helmintológico escolar em cinco estados das regiões Leste, Sul e Centro-Oeste. Ministério da Educação e Saúde, Departamento Nacional de Saúde, Divisão de Organização Sanitária. In: XI Congresso Brasileiro de Higiene. Curitiba, 1953.

11. Stephenson L, Latham M, Kinoti S, Kurz K, Brigham I. Improvements in physical fitness of Kenyan schoolboy infected with hookworm, Trichuris trichiura and Ascaris lumbricoides following a singles dose of albendazole. Transactions of the Royal Society of Tropical Medicine and Hygiene 84:277-282, 1990. 